\title{
High-Pressure Dynamic Strength of Materials
}

\author{
T. J. Vogler ${ }^{1}$. J. L. Brown ${ }^{2}$. C. A. Bronkhorst ${ }^{3}$
}

Published online: 7 May 2021

(c) Society for Experimental Mechanics, Inc 2021

Plasticity and the discovery of dislocations arose from studies of metals where pressure played a negligible role in the deformation. In contrast, early shock physics studies assumed that solids shocked to high pressures behaved as fluids without significant shear resistance. Eventually, though, signatures of elastic-plastic behavior were found in shock experiments, and efforts were made to measure and understand the strength (non-hydrostatic) behavior under high-pressure dynamic loading. In fact, material strength and processes such as dislocation motion and deformation twinning play an important role in a number of high-pressure applications including penetration and armor mechanics, planetary science, and inertial confinement fusion. This has led to a significant amount of research to improve our understanding of these phenomena.

A material's resistance to plastic deformation is, in general, quite complex. It can depend on factors such as crystallographic structure, microstructure, loading conditions (e.g., strain rate, stress, temperature), and loading history. Alloying and impurities as well as crystallographic and morphological texture are also important and can be used to tailor deformation resistance for performance. Developing an understanding of the multi-scale physics involved requires effective coupling of measurements, theory, and simulations that probe material behavior through a range of conditions and the length scales (e.g., atomic, dislocation, grain, polycrystal) involved.

With these considerations in mind, this special issue on High-Pressure Dynamic Strength has been assembled with contributions from academia, DoD laboratories, and DOE national laboratories. This issue provides a snapshot, albeit incomplete, of the state of the art in experiments and modeling of high-pressure strength from differing perspectives.

T. J. Vogler

tjvogle@sandia.gov

1 Sandia National Laboratories, 7011 East Ave., M.S. 9042, Livermore, CA 94550, USA

2 Sandia National Laboratories, Albuquerque, NM 87185, USA

3 University of Wisconsin-Madison, Madison, WI 53706, USA
Though most of the attention is given to metals, work on silica glass and explosives is also included.

In 2006, Vogler and Chhabildas (Int. J. Imp. Eng., 33, 812-825) reviewed six methods for determining high-pressure dynamic strength. Of those methods, this volume includes work with lateral stress gauges (Casem and Jordan), pressure-shear loading (Ravindran et al.), X-ray diffraction (Francom et al.), the growth of interface instabilities (Vogler and Hudspeth; Olles et al.), and the self-consistent approach (Brown et al.). Although these papers cover topics that are not new, the contributions herein reflect the significant advances made over the nearly a decade and a half since that review. Notably, the use of synchrotron X-rays for high-speed imaging and diffraction is a new development, while ramp (quasi-isentropic) loading and phase transformations have received increased attention.

The remaining papers cover a fairly wide range of topics, consistent with the broad range of ongoing research. Wang and Prakash study the behavior of magnesium at elevated temperature, while Reed et al. report results for tantalum obtained using a Lagrangian analysis approach. Dhiman et al. is an investigation of polymer bonded explosives. Two papers are focused on the development of and results from continuum scale models for inelastic deformation. Schill et al. develop a simple model to examine the effect of a phase transformation on dislocations in tin, while Clayton and Lloyd present a comprehensive macro-scale model for materials that include solid-solid phase transformations. Finally, Bourne suggests an important role for the weak shock limit, the pressure at which the plastic wave overtakes the elastic precursor, across a wide range of materials.

The guest editors gratefully acknowledge the technical contributions of all the authors and the reviewers. We would also like to thank former Editor-in-Chief Dr. Eric Brown for his early support of this project and Dr. Jennifer Jordan, current Editor-in-Chief of the Journal of Dynamic Behavior of Materials, for making this issue possible.

Publisher's Note Springer Nature remains neutral with regard to jurisdictional claims in published maps and institutional affiliations. 